\title{
THE END OF AN ERA
}

\author{
David Levy \\ London / England
}

\section{Background}

It had to come eventually. After 21 years of staving off the world's strongest computer programs I was ready for the inevitable, and the inevitable came. For those who might be slightly unaware of the background to this encounter I shall begin with a little computer-chess history.

It is fairly well known that in August 1968 I made a bet that I would not lose a match against a computer within 10 years (cf. ICCA Journal, Vol. 11, Nos 2/3, p. 99). When I won the bet in 1978, it was suggested that my victory would remove a target that many chess programmers had been aiming at. Why not leave the target in place? This idea came from Christopher Evans, a British scientist who wrote "The Mighty Micro" and produced a stimulating TV series on A.I. during the late 1970s. Unfortunately, Evans died shortly before his TV series was finished.

Chris Evans' idea was that I offer a prize for the first program to win a match against me, whenever it happened. At the time he was a contributing editor to Omni magazine, and his proposal was a 5,000 dollar prize, with $\$ 1,000$ coming from me and the balance of $\$ 4,000$ from Omni. I liked the idea of being the target for a few more years and accepted his suggestion immediately.

In 1984, I was challenged to a 4-game match by Cray Blitz, which I won easily. A later challenge from Cray Blitz could not be backed with suitable arrangements for playing another match, and a challenge from Hitech was withdrawn after I had accepted it. Another five years passed before I was put to the test again.

\section{The Match}

Late in 1988 Donald Michie, who was one of the original bettors twenty years earlier, suggested that he should organize a match between myself and Deep Thought. He was given approval for this venture by Omni, and found an excellent sponsor in the British company Infolink, which specializes in providing credit information. International Grandmaster Raymond Keene helped in the organization of the match and International Master Bob Wade, who has officiated as arbiter in World-Championship matches and many other top events, acted as the arbiter. The match was played at the London headquarters of the British Computer Society, with donations going to the Royal London Society for the Blind (we raised about 5,000 pounds for this excellent charity!). My opponent was linked by telephone from its home in Carnegie-Mellon University, and operated by Peter Jansen.

Prior to the match I realized that I was so rusty that I would need to train seriously if I was going to put up any kind of resistance. I had not competed seriously against humans for 11 years (my last outing was the 1978 Chess Olympiad in Buenos Aires). In 5-minutes games played in September against Jonathan Schaeffer and other friends I was utterly crushed. So in the month before the match I played in a few 5-minutes and Action Chess tournaments, just so that I could begin to remember how to move the pieces properly. The most important part of my pre-match training was 3 days spent with my second, International Master Danny Kopec, who flew into London specially to help me. Danny had worked with me for the Cray Blitz match in 1984 and is an excellent second, being a stronger chess-player than me as well as someone who understands computers. We played a large number of 7-minute games to tone up my mental reflexes and we studied various openings ideas. I think that Danny was able to improve my playing strength by about 200 points in those 3 days, and I felt very pleased at the effect his help had on my morale and on my ability to think like a chess-player again. Unfortunately, it turned out that the layers of rust were so thick, that even Danny's help was not enough.

Before the match I heard that William Hill, the British bookmakers, were making me favourite and offering odds of 3 to 1 against the computer. I was telling my closest friends that I expected to lose 4-0, an easy estimate for me to make because I knew how rusty I was and I knew the strength of my opponent. 


\section{Game 1}

\section{David Levy - Deep Thought}

I entered the first game, in which I had White, with some hope that I could get the upper hand in the opening and hang on to my advantage until the endgame, but my illusions were soon shattered when I overlooked various "obvious" moves and got flattened, after having had a promising position. My $4^{\text {th }}$ move, 4. e3, has the rather unsubtle point that 4... gxh4 allows 5. Qh5 mate! This is why White can afford to get the Bishop "trapped" by 3 .... g5.

One of my major errors in this game was in not playing h4. After $8 . \mathrm{h} 4 \mathrm{~g} 4 \mathrm{9}$. Ne2, for example, the Knight comes to 4 and Black's King's side will be under pressure for a long time to come.

The game started to go downhill with the plan initiated by my $11^{\text {th }}$ move Bf2. I had intended to meet 11 ... e 5 with 12. f4, but when the opportunity arose I noticed 12... exf4, and if 13. Qxh5 fxe3 14. Bxe3 Qe7. But even stronger would have been the move that Deep Thought intended: (11... e5 12. f4) 12. ... Nxf4!! 13. exf4 e4, followed by 14. ... gxf4, when Black regains the piece with a great game. When I played 12. Qb3 I believed that 12. ... $\mathrm{f} 4$ was bad because I overlooked the strength of 15 ... Na5. From then on the game was a massacre.

1. d4 f5 2. Bg5 h6 3. Bh4 g5 4. e3 Nf6 5. Bg3 d6 6. c3 Bg7 7. Nd2 O-O 8. f3 Nc6 9. Bc4+ d5 10. Bd3 Nh5 11. Bf2 e5 12. Qb3 f4 13. e4 exd4 14. cxd4 Bxd4 15. exd5 Na5 16. Qc2 Bxf2+ 17. Kxf2 Qxd5 18. Ne2 Bf5 19. Ne4 Qc6 20. N2c3 Qb6+ 21. Kf1 Rad8 22. Rd1 Rfe8 23. Be2 Rxd1+ 24. Qxd1 Bxe4 25. Nxe4 Nf6 26. Qa4 Re5 27. Qd1 Nd5 28. Qa4 Qxb2 29. Qd7 Qc1+ 30. Kf2 Qxh1 31. Qd8+ Kg7 32. Qd7+ Ne7 33. Qxc7 Nac6 34. Nd6 Rxe2+ 35. Kxe2 Qxg2+36. Ke1 Qh1+ White resigns.

\section{Game 2}

Deep Thought - David Levy

I struggled for a long time in an inferior position, putting up quite a lot of resistance, but then I made a horrible decision (which may not have affected the outcome, as it happens). On move 30 I should have played $30 . .$. $\mathrm{Kg7}$. I rejected this because I felt that after 31. Ne8+ Kf8 32. Nd6, White's minor pieces are congregating nicely on the Q-side and I will probably soon lose a Pawn. Indeed, Deep Thought assessed its position as being roughly a Pawn ahead at this point. I also made the mistake of thinking that after 30 ... Bxf6 31. exf6, I might be able to win the f6 Pawn in some variations. I actually saw the whole of the variation which followed, up to the point where I played 36. ... Na4, but I completely missed the fact that after the exchange of b-Pawns at move 37 the computer could end the game with 38. $\mathrm{Ng} 4$. At this point Deep Thought announced mate in 12 , though I was not told about this and struggled on for a few moves. (Bent Larsen once said that a chess-player should not resign until the spectators can understand why.)

1. c4 d6 2. Nc3 g6 3. d4 Bg7 4. e4 d6 5. Be3 Nf6 6. Be2 O-O 7. f4 c6 8. e5 Ne8 9. Nf3 d5 10. O-O Nc7 11. Rc1 e6 12. Qe1 b5 13. cxd5 cxd5 14. Nd1 Ra7 15. Nf2 Nd7 16. Qa5 Na8 17. Qa3 Qb6 18. Bd2 a5 19. Qd6 b4 20. Rc6 Qd8 21. Rfc1 Bb7 22. R6c2 Ndb6 23. Qxd8 Rxd8 24. Be3 Rc8 25. Rc5 Bf8 26. Bd3 Rd8 27. R5c2 Rc8 28. Rxc8 Bxc8 29. Ng4 Be7 30. Nf6+ Bxf6 31. exf6 Rc7 32. Ne5 Rxc1+ 33. Bxc1 Bb7 34. a3 Nc7 35 . axb4 axb4 36. Bd2 Na4 37. Bxb4 Nxb2 38. Ng4 e5 39. Nh6+ Kh8 40. Nxf7+ Kg8 41. Nh6+ Kh8 42. f5 Black resigns.

\section{Game 3 \\ David Levy - Deep Thought}

The point of Black's strange looking second move is that it threatens to win material in some variations. For example (2. ... c6 3. c3 h6) 4. Bh4?? Qb6 (threatening ... Qxb2) followed by 5. ... g5, trapping the Bishop. The point is that ... Qb6 not only attacks b2, it also creates a bolt hole for the black King, so that White's Qh5 will only be check, not mate.

I had a definite plus after move five, but when playing 6. e4 I overlooked the move 7... Nh7, which cost me a piece for 2 Pawns. Subsequently I failed to make the most of my attacking chances, for example 8 . Nc4 is inferior to both 8. Qh5+ and 8. Bg3 f4 9. Bd3 (with the idea of 9. ... fxg3 10. Qc2) 9. ... Bg7. After the game Peter Jansen told me that had I played 8. Qh5+Deep Thought would have been back in book!!! 
1. d4 f5 2. Bg5 c6 3. c3 h6 4. Bf4 Nf6 5. Nd2 d6 6. e4 g5 7. e5 Nh7 8. Nc4 gxf4 9. Qh5+ Kd7 10. Qxf5+ Kc7 11. Qxf4 Be6 12. Nf3 Rg8 13. Ne3 Ng5 14. exd6 exd6 15. d5 Bxd5 16. Nxd5 cxd5 17. Nd4 Qe7+ 18. Be2 Nc6 19. Nb5+ Kb8 20. h4 Ne6 21. Qf3 Ne5 22. Qxd5 Nf4 White resigns.

\section{Game 4 \\ Deep Thought - David Levy}

I was continually surprised at the excellent positional moves coming from Deep Thought. Whenever I had an idea, my opponent forestalled it. Later I learned from Peter Jansen that 30 new terms had been added to the program's evaluation function following the match against Kasparov which had taken place a few weeks earlier.

After being outplayed completely in the opening and early middle-game, I blundered away a piece.

1. c4 d6 2. Nc3 Nd7 3. d4 g6 4. Nf3 Bg7 5. e4 c5 6. Be2 cxd4 7. Nxd4 Ngf6 8. Be3 O-O 9. O-O a6 10. f3 Re8 11. Qd2 Ne5 13. h3 Bd7 14. Nf3 Qa5 15. a3 Rad8 16. b4 Qc7 17. Rac1 b6 18. Bd3 Qb7 19. Qf2 Rb8 20. e5 Nh5 21. b5 axb5 22. cxb5 Nd8 23. g4 Bh8 24. gxh5 Bxh3 25. hxg6 hxg6 26. Rfd1 Qd7 27. Ng5 Bg4 28. Qh4 $\mathrm{Bg} 7$ 29. Rd2 Bh5 30. Nd5 Qa7 31. Rc7 Rb7 32. exd6 exd6 33. Rc8 Qxa3 34. Ne4 Black resigns.

\section{No excuses}

Despite the 4-0 score I actually enjoyed playing the match. Deep Thought is a very interesting opponent with a delightfully pragmatic style of play. "Very American", as the Russians used to say of Bobby Fischer. I do not have the usual stock of excuses: I was not feeling particularly unwell; I was not sitting too close to the heater; there was no disturbing noise outside the playing room; the lighting was not poor; the computer operator, Peter Jansen, was not smoking. The reason for the score is simple to explain: an IM who has not played serious chess for 11 years is no match for the enormous power of Deep Thought. In fact I do not believe that there is an International Master in the world today who, having not played a single serious game for 10 years or more, could avoid losing a match to Deep Thought. It is true that my understanding of computer chess closed the gulf between us by 100 or 150 rating points, but the gulf was too wide to start with.

\section{Congratulations}

I would like to congratulate the Deep Thought team on winning the Omni Prize. They fully deserve it and I am sure they will go on to great things in computer chess. I have not revised my estimate of 20-25 years before one of Deep Thought's heirs takes the world crown from Kasparov or his successor, but I do believe that Deep Thought could reach the super-Grandmaster class (2600 Elo rating) within 5 years.

As to beating Kasparov by 1995 - no way! Do you want to make a bet on it?

P.S. When I saw Deep Thought's games in which Karpov was rather lucky to win (see this Journal, pp. 37-40), and when Grandmaster Pfleger made "only" two draws in a two-game match (see this Journal, p. 40), I felt that my wipeout did not look so bad after all.

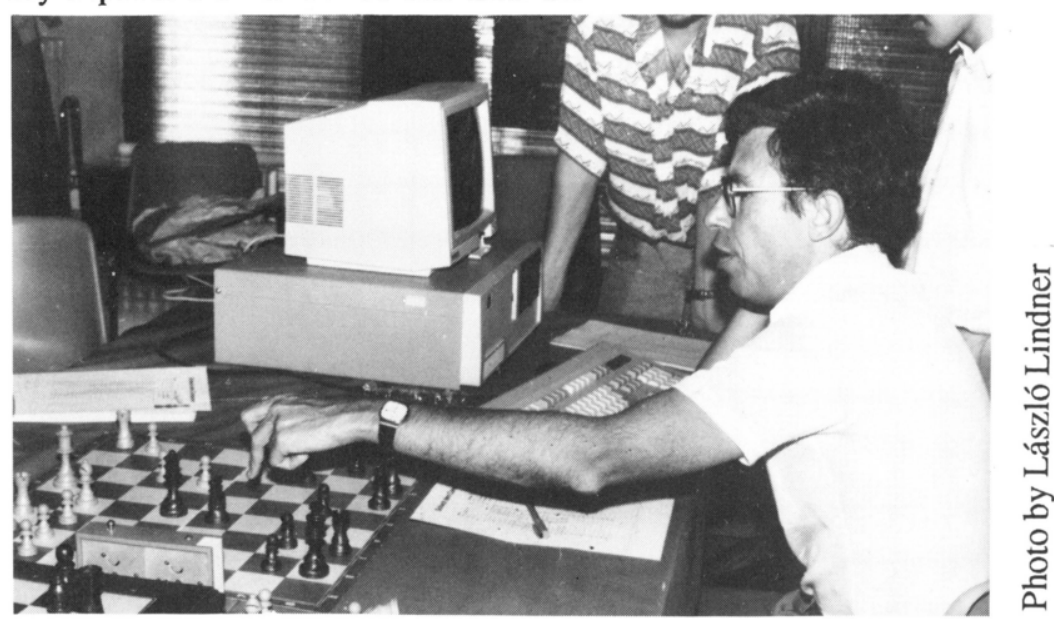

